\title{
In Vivo Expression of Interleukin-37 Reduces Local and Systemic Inflammation in Concanavalin A-Induced Hepatitis
}

\author{
Ana-Maria Bulau, ${ }^{1}$ Michaela Fink, ${ }^{1}$ Christof Maucksch, ${ }^{1}$ Roland Kappler, ${ }^{2}$ Doris Mayr, ${ }^{3}$ \\ Kai Wagner, ${ }^{3}$ and Philip Bufler ${ }^{1}$ \\ ${ }^{1}$ Department of Pediatrics, Dr. von Hauner Children's Hospital, \\ Ludwig-Maximilians-University, 80337 Munich, Germany \\ ${ }^{2}$ Department of Pediatric Surgery, Dr. von Hauner Children's Hospital, \\ Ludwig-Maximilians-University, 80337 Munich, Germany \\ ${ }^{3}$ Institute of Pathology, Ludwig-Maximilians-University, 80337 Munich, Germany
}

Received 15 July 2011; Accepted 7 November 2011

Academic Editor: Giamila Fantuzzi

We recently reported that after LPS stimulation, IL-37 translocates to the nucleus and reduces the expression of proinflammatory cytokines. The aim of this study was to investigate whether transiently expressed IL-37 in mice reduces inflammation in concanavalin A (ConA)-induced hepatitis and LPS-induced sepsis. Transgene IL-37 expression was detected in the liver lysate of mice injected with IL-37 plasmid-DNA after hydrodynamic tail vein injection. All mice developed severe acute hepatitis after ConA injection. No difference in the histological score and serum ALT was observed between the two groups that might be explained by patchy expression of IL-37 protein in the liver. However, 2 hrs after ConA injection, serum levels for IL- $1 \alpha$, IL-6, IL-5, and IL-9 were significantly reduced in IL-37-expressing mice as seen for the LPS model. In conclusion, in vivo expression of human IL-37 in mice reduces local and systemic inflammation in ConA-induced hepatitis and LPS challenge.

KEYWORDS: IL-37, cytokines, concanavalin A, hepatitis, inflammation, lipopolysaccharide 


\section{INTRODUCTION}

The IL-1 family of cytokines possesses a variety of immunoregulatory properties in response to infection and inflammation [1]. Most of the 11 known members of the IL-1 family share common cellular receptors either acting as agonists or antagonists. The most prominent receptor antagonist is IL-1Ra. IL-37 (IL-1F7), IL-33 (IL-1F11), and IL-1 $\alpha$ were shown to translocate to the nucleus after cell stimulation and might also act as transcriptional modulators. We recently showed that IL-37 stands apart being emerged as a fundamental anti-inflammatory cytokine [2]. Five different splice variants of IL-37 have been described [3-6]. IL-37 binds to the IL-18R $\alpha[7,8]$ without having an agonistic or antagonistic function at the receptor level [7, 9]. IL-37 protein is expressed in human monocytes, and it is upregulated by a variety of TLR ligands [2, 10].

Intravenous injection of ConA into mice induces T-cell-mediated liver injury, which is characterized by rapidly increased serum aminotransferase and cytokine levels, leukocyte infiltration of the liver, and hepatocyte necrosis $[11,12]$. Administration of ConA in mice leads to an acute, partly apoptotic hepatic injury that is subsequently overlaid by massive necrosis $[11,13]$. T-cell activation, that is, hepatic natural killer T (NKT) cells, were shown to play a critical role in ConA-induced liver injury $[11,14]$ by releasing a variety of cytokines, including interleukin 4 (IL-4), IL-5, interferon gamma (IFN- $\gamma$ ), and tumor necrosis factor alpha (TNF- $\alpha$ ) [15-17]. Activation of CD4+ T cells in ConA-induced hepatitis results in the release of IL-1 $\beta$, IL-2, IL-4 [18], tumor necrosis factor- $\alpha$ (TNF- $\alpha)$ [13], and IFN- $\gamma$ [19], the last two playing a critical role in disease development as proven by antibody treatment [13, 19].

Activated $\mathrm{T}$ cells also play an important role in tissue repair after liver injury by producing antiinflammatory cytokines such as IL-10 and antiapoptotic cytokines such as IL-6 [20, 21]. Protection of hepatitis can be induced by the administration of recombinant IL-6 if applied before ConA application [22]. IL-22 has also been shown to play a protective role in hepatitis [23].

To gain a better understanding of immune-mediated hepatitis and to provide further insight in the physiological function of IL-37, we applied the model of ConA-induced hepatitis in mice transiently expressing human IL-37 after hydrodynamic tail vein injection of plasmid-DNA. We also employed LPSinduced shock to test whether transient expression of IL-37 in extralymphatic tissue is similarly effective to reduce inflammation as shown for transgenic mice [2].

\section{MATERIALS AND METHODS}

\subsection{Chemicals}

All reagents were purchased from Sigma-Aldrich GmbH (Germany) unless indicated.

\subsection{Plasmid Construction}

Human IL-37 cDNA was cloned into the expression plasmid pTarget, which contains a constitutively active CMV promotor, as previously described [10]. All plasmids were isolated by "low LPS" MaxiPrep kit (Qiagen, Germany) to reduce nonspecific inflammation by contaminating LPS.

\subsection{In Vivo Expression of IL-37}

Animal protocols were approved by the Federal Government of Bavaria, Germany. Six to eight weeks old, female C57/BL/6 mice were purchased from Janvier (France). The animals were housed at controlled temperature with light-dark cycles, with free access to food and water and were acclimatized before being studied. For in vivo expression of human IL-37, mice were rapidly injected with either $20 \mu \mathrm{g}$ of empty pTarget or pTarget-IL-37 in $2 \mathrm{~mL}$ of Ringer's solution into the tail vein ("hydrodynamic injection" [24]). The plasmid pLuc was coinjected at a ratio of $1: 20$ for in vivo transfection control. 


\subsection{Animal Models and In Vivo Imaging}

$48 \mathrm{hrs}$ after plasmid-DNA injection, ConA $(200 \mu \mathrm{g})$ in pyrogen-free saline was injected into the tail vein of mice to induce hepatitis. Alternatively, $10 \mu \mathrm{g}$ of LPS (E. coli 055:B59) was injected intraperitoneally. $2 \mathrm{hrs}$ after LPS injection, mice were anesthetized by isoflurane, blood was taken by intracardiac puncture, and mice were sacrificed. $2 \mathrm{hrs}$ after ConA injection, a blood sample was taken for cytokine measurement from the retroorbital plexus under isoflurane anesthesia. $24 \mathrm{hrs}$ after ConA injection, in vivo bioluminescent imaging was performed as previously described [25]. The substrate luciferin was injected into the intraperitoneal cavity at a dose of $3 \mathrm{mg}$ in aqueous solution $10 \mathrm{~min}$ before imaging. Ventral images were collected for $1 \mathrm{~s}$ using the IVIS imaging system (Xenogen Corp., Alameda, CA, USA). Photons emitted from the liver region were quantified using Living Image software (Xenogen Corp.). Another blood sample was then obtained, and the mice were subsequently sacrificed. The livers were removed and stored for histological, protein, and cytokine analysis.

\subsection{Western Blot}

Frozen livers were sonicated in phosphate-buffered saline containing $0.1 \%$ Tween 20 and protease inhibitors. After centrifugation (13,000 rpm, $\left.7 \mathrm{~min}, 4^{\circ} \mathrm{C}\right)$, supernatants were aspirated, and their protein concentrations were determined. $90 \mu \mathrm{g}$ of liver lysates were separated on a 10\% SDS polyacrylamide gel and transferred on to a nitrocellulose membrane (Hybond ECL, Amersham Biosciences, Freiburg, Germany). For detection of IL-37 protein in the liver lysates, a mouse monoclonal Ab was used [10]. After washing, the membrane was incubated with a secondary horse-radish-labeled antibody (Sigma-Aldrich) at room temperature for $1 \mathrm{~h}$. The membranes were developed with enhanced chemiluminescence (SuperSignal, Pierce, Rockford, IL, USA).

\subsection{Analysis of Liver Injury}

The extent of liver injury was assessed $24 \mathrm{hrs}$ after ConA administration by determination of serum alanine aminotransferase (ALT) using a Hitachi 917 Analyzer (Hitachi).

\subsection{Liver Histology and Immunohistochemistry}

A segment from the right lobe of the liver was fixed in $10 \%$ formalin, dehydrated in ethanol, and paraffinembedded for histologic analysis. $5 \mu \mathrm{m}$ sections were stained with hematoxylin and eosin and reviewed by a single, blinded pathologist. A histological score was performed as previously described [26]. For lobular inflammation, no inflammation was counted as 0 , mild lobular inflammation $(<10 \%$ of liver parenchyma) as 1, moderate lobular inflammation (10-50\% of liver parenchyma) as 2, and a score of 3 was given for severe lobular inflammation ( $>50 \%$ of liver parenchyma). For portal inflammation, no portal inflammation was counted as 0 , mild portal inflammation $(<1 / 3$ of portal tracts $)$ as 1 , moderate portal inflammation (approximately $1 / 2$ of portal tracts) as 2, and a score of 3 was given for severe portal inflammation ( $>2 / 3$ of portal tracts). The scores for portal and lobular inflammation were added, representing the inflammation score. For necrosis, no necrosis was counted as 0 , necrosis of $<10 \%$ of liver parenchymas 1 , necrosis of 10-25\% of liver parenchyma as 2, and a score of 3 was given for necrosis of $>25 \%$ of liver parenchyma. Inflammation and necrosis scores were added resulting in the total histological score.

Immunostaining against IL-37 in formalin-fixed liver tissue was performed with a polyclonal antibody against IL-37 (anti-human IL-1F7/FIL1 zeta goat IgG R\&D-Systems) and a mouse monoclonal $\mathrm{Ab}$ [10]. The mouse antibody was not as sensitive with the immune staining as the polyclonal goat antibody but similarly specific. The final immune stainings were, therefore, done with the polyclonal goat antibody. 


\subsection{Measurement of Cytokines}

Levels of serum cytokines in LPS- or ConA-treated mice as well as liver lysates were measured by Bio-Plex multiarray cytokine assay (Bio-Plex Multiarray Cytokine Assay, Bio-Rad Laboratories GmbH, Munich, Germany).

\subsection{Statistical Analysis}

Results are expressed as mean \pm SEM. Differences between control and treated groups were compared by Mann-Whitney- $U$-test. Statistical analysis was performed with Prism 5 Version 5.0d for Macintosh.

\section{RESULTS}

\subsection{In Vivo Luciferase Activity in the Liver of C57/BI6 Mice after Hydrodynamic Tail Vein Injection of Plasmid-DNA}

Anatomic distribution of luciferase protein, coexpressed with IL-37, was investigated by in vivo imaging. The substrate luciferin was intraperitoneally applied into DNA-injected C57/BL/6 mice after ConA application shortly before imaging analysis. In vivo measurement of luciferase activity did not differ between IL-37-expressing mice and control mice showing high expression in liver region (Figures 1(a) and $1(\mathrm{~b}))$.

\subsection{High Transgene IL-37 Expression in Liver after Hydrodynamic Tail Vein Injection of Plasmid-DNA}

In order to monitor the expression of IL-37, tail-vein-injected C57/BL/6 mice were sacrificed after luciferase measurement, and the level of IL-37 protein was assayed in liver lysate by Western blotting. High level of transgene IL-37 expression was detected in liver lysate of LPS or ConA-treated mice after tail vein injection of pTarget-IL-37 plasmid-DNA, while mice injected with control plasmid (empty pTarget) did not express human IL-37 in the liver (Figure 1(c)). In addition, immunohistochemistry showed positive staining of the liver of IL-37 expressing but not control mice (Figure 2). Expression of transgene IL-37 in the liver was not homogenous but showed a patchy distribution.

\subsection{LPS-Induced Inflammation Is Reduced in IL-37-Expressing Mice}

Shock produced by bacterial lipopolysaccharides (LPSs) induces a systemic cytokine response as well as liver injury in mice [27]. To see whether transient IL-37 expression in the liver of mice can attenuate systemic inflammation, we analyzed serum cytokine levels $2 \mathrm{hrs}$ after LPS injection (Figure 3). The T-cellderived cytokines IFN- $\gamma$, IL-2, and IL-4 and the macrophage-derived cytokine IL-6 and IL-9 expression were significantly reduced in IL-37-expressing mice when compared to controls after LPS challenge. Expression of proinflammatory cytokines IL-12 and IL-5 was also significantly reduced in the plasma from IL-37 expressing mice after LPS challenge. In contrast, serum levels of the anti-inflammatory cytokine IL-10 were unaffected in mice expressing IL-37 compared to control mice.

\subsection{IL-37 Expression in Mice Reduces ConA-Induced Liver Inflammation}

After we showed that transient expression of IL-37 in mice reduces systemic inflammation after LPS challenge we wanted to investigate the functional relevance of IL-37 in another model of inflammation. ConA induced hepatitis is a well-established model of systemic and local inflammation which is associated with the production of various cytokines and liver damage. ConA injection induced high serum alanine aminotransferase (ALT) levels $24 \mathrm{hrs}$ after induction of hepatitis in both IL-37-expressing and control mice. 

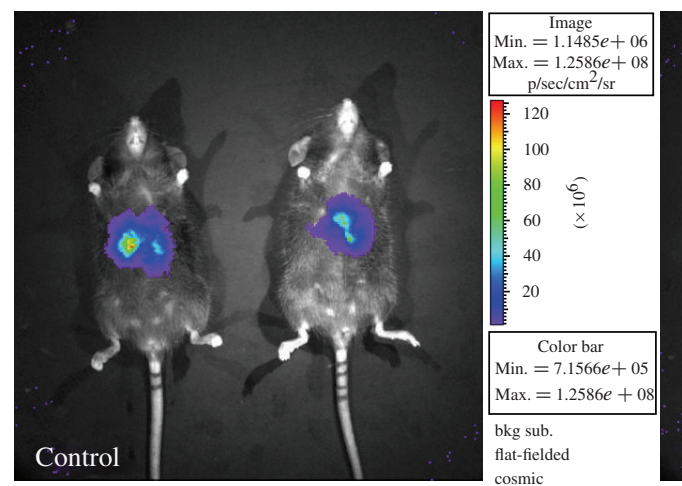

Click no. CR20080725185405 Series: pTarget empty Fri., Jul 25, 2008 18:54:16 Experiment: ConA Bin: $\mathrm{M}(8)$, Fov15, f1, $1 \mathrm{~s}$

Label: pTarget

Filter: open

Comment: $72 \mathrm{~h}$ after DNA-injection, Camera: IVIS 13224, S1620EEV Analysis comment

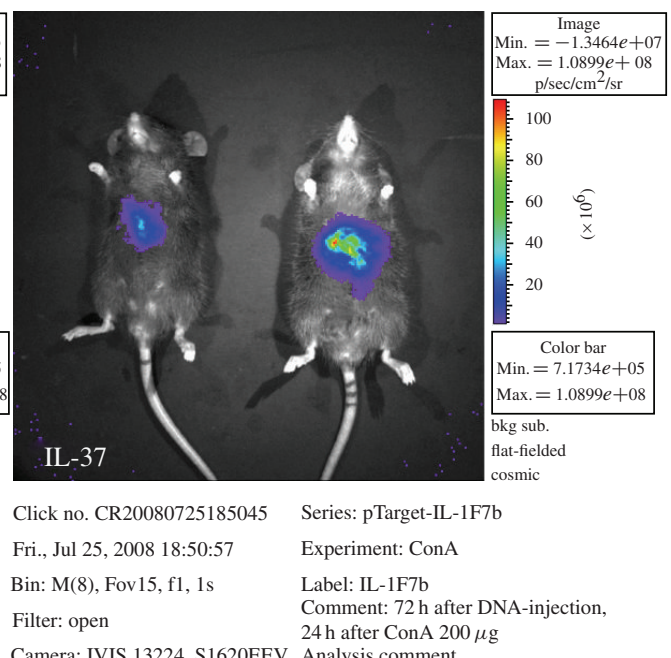

Camera: IVIS 13224, S1620EEV Analysis comment

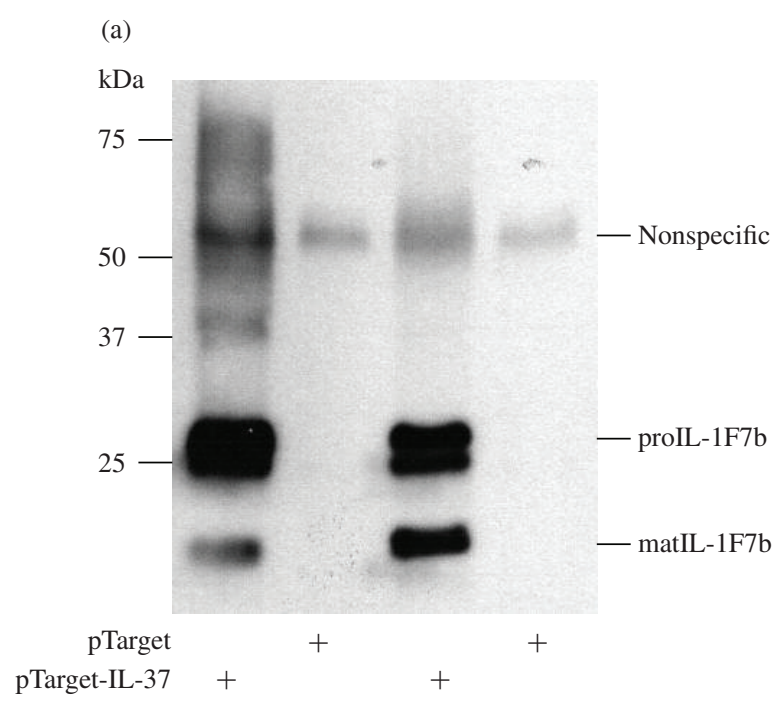

(b)

(c)

FIGURE 1: In vivo luciferase activity in the liver of C57/BL/6 mice after hydrodynamic tail vein injection of plasmid-DNA. C57/BI6 mice were rapidly injected with pTarget-IL-37 $(20 \mu \mathrm{g})$ and pLuc plasmid-DNA $(1 \mu \mathrm{g})$ in $2 \mathrm{~mL}$ of Ringer's solution. After $48 \mathrm{hrs}$ concanavalin A $200 \mu \mathrm{g}$ was applied into the tail vein. $24 \mathrm{hrs}$ after ConA injection, luciferase expression was measured in vivo using the IVIS imaging system (a). Luciferase activity in IL-37-expressing mice (open bar) and control mice (closed bars) (b). IL-37 protein detected in liver lysate of mice injected with IL-37-pTarget plasmid mice and control mice (empty pTarget) by Western blotting (c).

Hydrodynamic tail vein injection itself did not induce liver damage as assessed by ALT measurement of control mice (Figure 4). Serum levels of IL-1 $\alpha$, IL-5, IL-6, and IL-9 were significantly reduced $2 \mathrm{hrs}$ after ConA injection in mice expressing IL-37 (Figure 5(a)). This effect was not sustained, since $24 \mathrm{hrs}$ after induction of hepatitis, there was no difference in serum cytokine expression between the two groups of mice (Figure 5(a)). Serum levels of IL-1 $\beta$, IL-2, IL-3, IL-4, IL-10, IL-12 (p40 and p70), IL-13, IL-17, Eotaxin, G-CSF, GM-CSF, IFN $\gamma$, KC, MCP-1, MIP- $1 \alpha$, MIP- $1 \beta$, Rantes, and TNF $\alpha$ were not significantly different (data not shown).

Consistent with equal ALT concentrations, histologic evaluation of the liver showed a similar extent of liver injury in both IL-37 transgenic mice and control mice after ConA injection. Treatment with ConA 

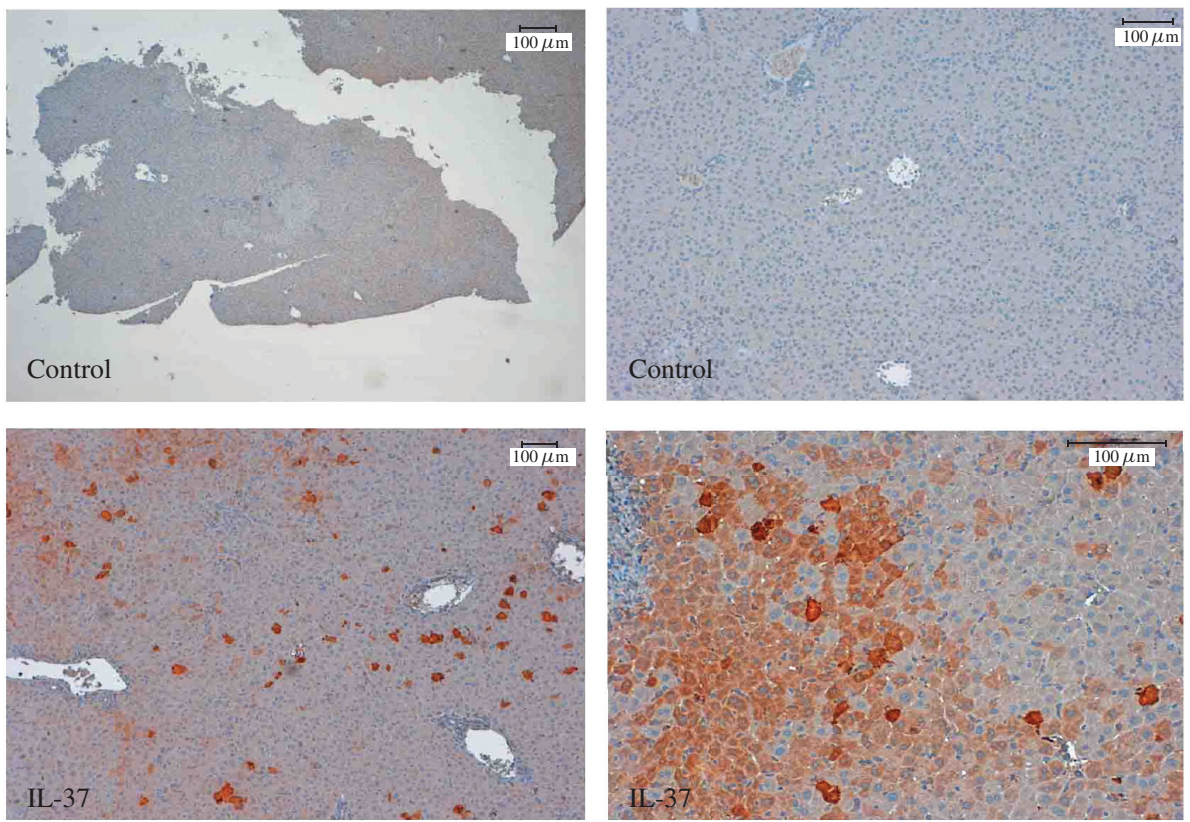

FIGURE 2: Detection of transgene IL-37 by immunohistochemistry. $5 \mu \mathrm{m}$ sections of mouse livers after plasmid-DNA injection with control plasmid (empty pTarget) or IL-37-pTarget were stained with a polyclonal antibody against IL-37.

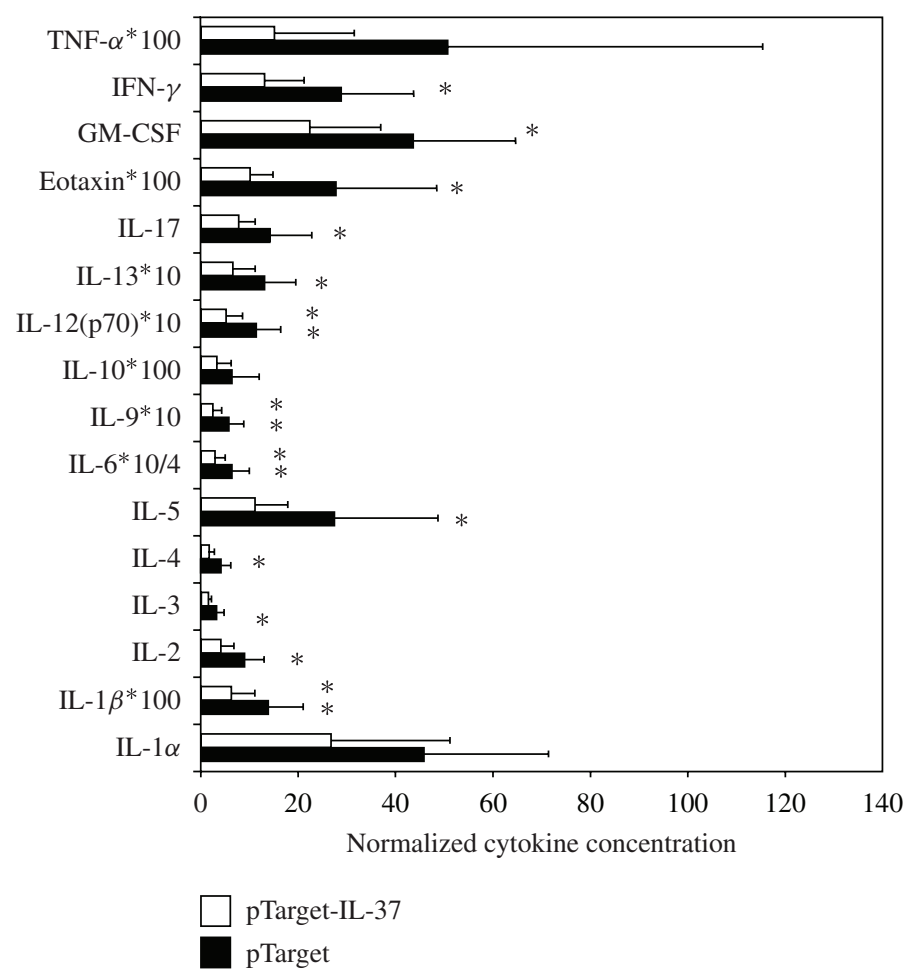

FIGURE 3: LPS-induced serum cytokines in mice expressing IL-37. Tail vein injected C57/BI6 mice (with or without IL-1F7b plasmid-DNA) were challenged with LPS $(10 \mu \mathrm{g})$ intraperintoneally $(n=7-9)$. After 2 hrs the mice were sacrificed and serum cytokines were measured by a multiarray cytokine assay as described. 


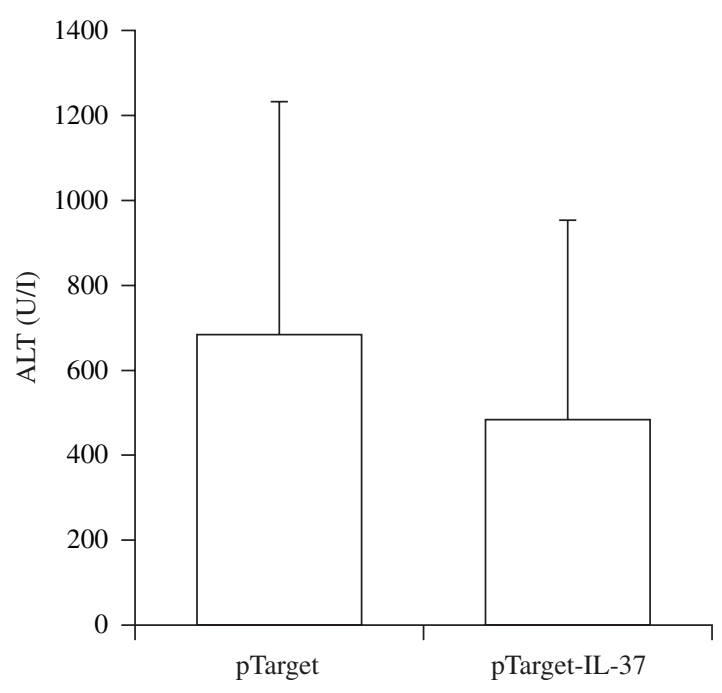

FIGURE 4: Serum ALT levels in ConA-induced hepatitis. Serum ALT was measured in tail-vein-injected C57/BI6 mice (with or without IL-1F7b cDNA, $n=3-4$ ) $24 \mathrm{~h}$ after ConA treatment. Serum ALT levels were measured by a Hitachi 917 Analyzer.
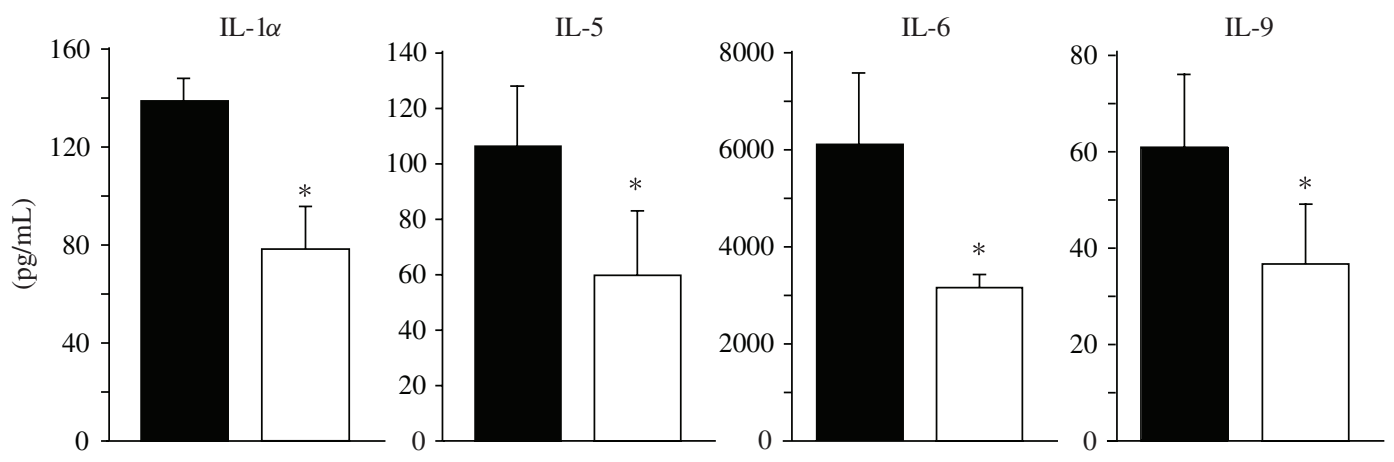

(a)
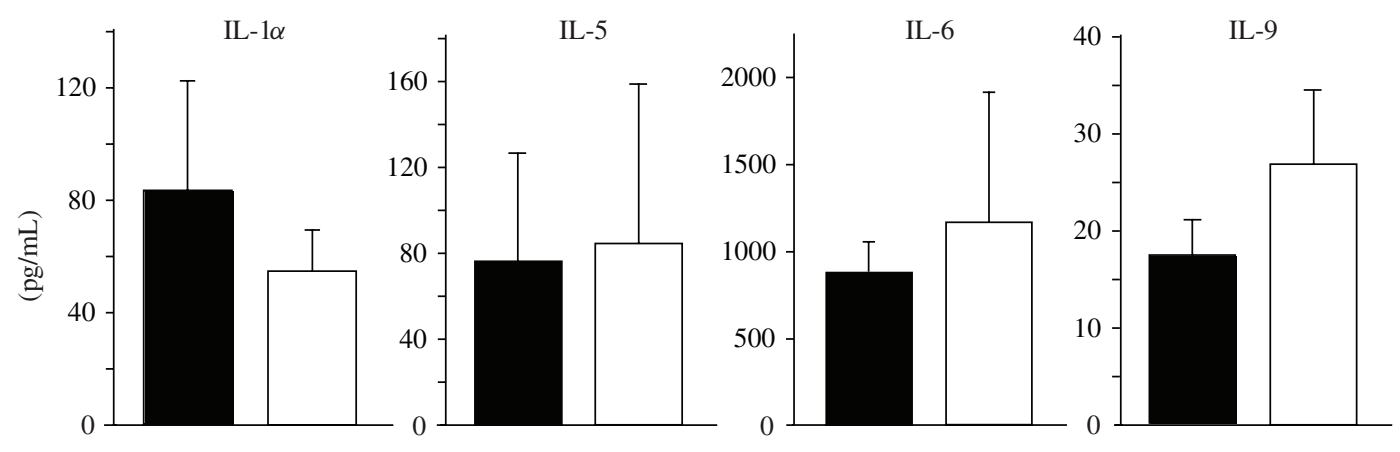

pTarget

(b)

pTarget-IL-37

FIGURE 5: Expression of serum cytokines in IL-37-expressing mice after ConA-induced hepatitis. Serum cytokines were measured by a multiarray cytokine assay $2 \mathrm{hrs}$ (a) and $24 \mathrm{hrs}$ (b) after ConA treatment in IL-37 expressing mice (open bars, $n=4$ ) and control mice (closed bars, $n=3$ ). 


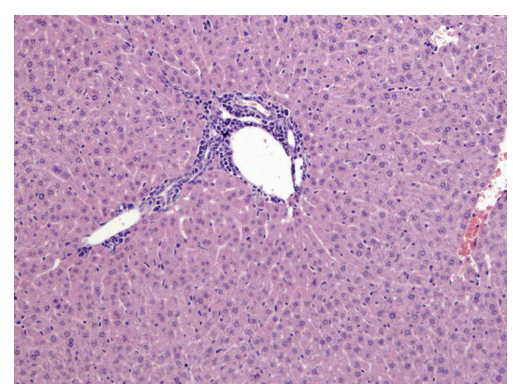

(a)

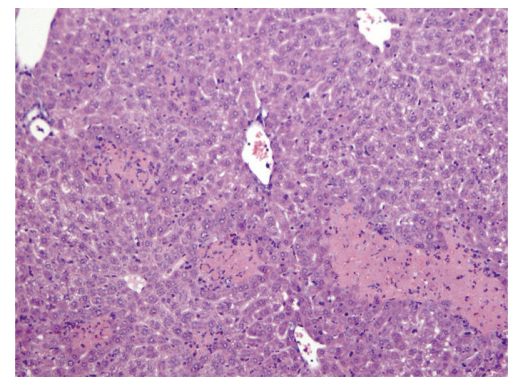

(c)

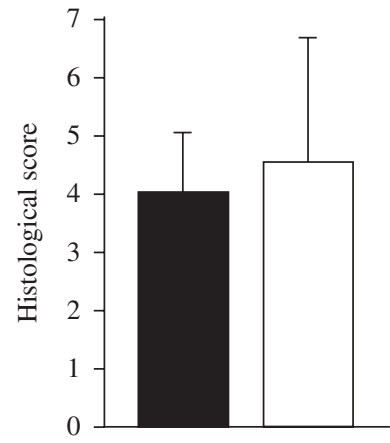

(e)

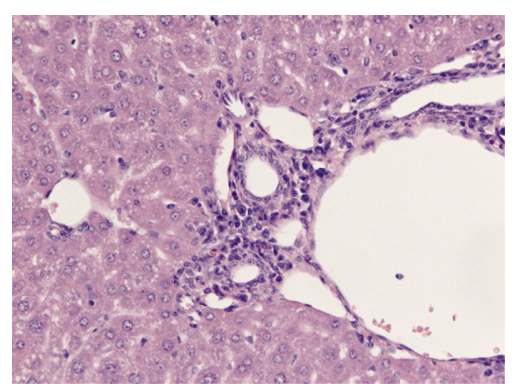

(b)

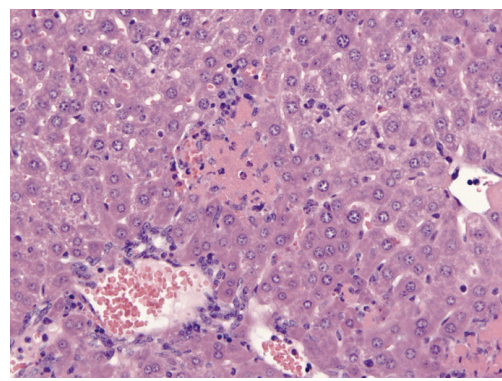

(d)

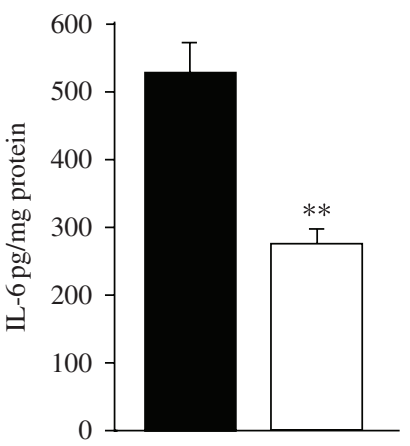

(f)

FIGURE 6: Histological hepatitis score and IL-6 protein expression in the liver of mice after ConA-induced hepatitis. Areas of portal inflammation ( $a$ and b) and necrosis ( $c$ and $d$ ) in ConA-induced hepatitis in control mice (histology of IL-37-expressing mice not shown). Histological hepatitis score analyzed for IL37 expressing mice (open bars) and control mice (closed bars) (e). IL-6 in liver lysate was analyzed by multiarray cytokine assay and is expressed as $\mathrm{pg} / \mathrm{mg}$ total protein in liver lysate (f).

induced marked lobular and portal inflammation (Figures 6(a) and 6(b)) as well as hepatocyte necrosis (Figures 6(c) and 6(d)). Hydrodynamic injection of plasmid-DNA itself did not induce liver damage as assessed by serum ALT (see Figure 4, histology not shown). In both groups, the histologic score was similar $(4.5 \pm 2.1$ versus $4.0 \pm 1.0)$ (Figure 6(e)). However, IL-6 in the liver lysate was reduced in IL-37 expressing mice after ConA-induced hepatic injury indicating a downregulation of local inflammation in the liver by transiently expressed IL-37 (Figure 6(f)).

\section{DISCUSSION}

We have recently reported that IL-37 transgenic mice are protected against LPS-induced shock, and in vivo silencing of endogenous Smad3 significantly reduced the anti-inflammatory properties of IL-37 [2]. Accumulated data reveal IL-37 as a natural modulator of inflammation acting in a feedback mechanism to 
overcome an exacerbate immune reaction. To address whether IL-37 could be a therapeutic target for other inflammatory diseases, we employed ConA-induced hepatitis as a model for this study.

The IL-1 family is very unique in that there is no species specificity. IL-37 is the only IL-1 family member of which the mouse homolog is not detected yet. Overexpression of IL-37 in a variety of murine and human cell lines resulted in a marked decrease of cytokine expression after LPS $[2,28]$ indicating that IL-37 is active in both the human and murine system.

We introduced a plasmid that expresses human IL-37 into mice by the hydrodynamic-based gene delivery procedure, a method that has previously been proved with high efficiency for delivery of genes into the mouse liver $[29,30]$. This mode of in vivo protein expression might be advantageous compared to transgenic animals since nonspecific effects by nondirected integration of the transgene into the genome are excluded. Indeed, the hydrodynamic tail vein procedure successfully delivered IL-37 into mouse livers without organ damage as observed by in vivo measurement of luciferase activity which did not differ between IL-37 expressing mice and control mice. Detection of IL-37 by western blotting showed high levels of transgene IL-37 expression in liver lysate. Immunohistochemistry staining showed a patchy distribution of overexpressed IL-37 in the liver.

Recently, we showed experimental data indicating that IL-37 transgenic mice are protected against nonlethal LPS-induced shock by shifting the cytokine equilibrium away from excessive inflammation. Here, we similarly observed suppression of proinflammatory cytokine but not anti-inflammatory IL-10 secretion in serum from mice expressing IL-37 compared to controls. The hydrodynamic tail vein injection delivers the transgene mainly into the liver. We found transgene IL-37 being predominantly expressed in the hepatocytes and not the immune cells of the liver (data not shown). Therefore, our results indicate that expression of IL-37 in extralymphatic tissues as the liver can modulate systemic inflammation induced by LPS. This might be related to the action of IL-37 released into the extracellular space or the modulation of the hepatocyte inflammatory response.

To see whether the protective effect of IL-37 is expanding beyond the LPS model of systemic inflammation, we employed a mouse model of experimental hepatitis induced by ConA [13]. Administration of ConA to mice results in T-cell and macrophage activation that is characterized by a rapid increase of proinflammatory cytokines in plasma and liver tissue [6, 11,31]. Mice intravenously injected with ConA were shown to suffer from acute liver failure with signs of injury restricted to the liver and an increase of serum transaminases [11]. In our study, transient IL-37 expression could not inhibit ConA-induced liver necrosis as manifested by similar serum levels of ALT and histological severity score after ConA insult in both IL-37expressing and control mice. This might be in part explained by the patchy distribution of transgene IL-37 after hydrodynamic tail vein injection. In contrast, at the serum cytokine level, we observed a protective effect of ectopically expressed IL-37. This effect was not sustained, since 24 hrs after ConA application, no significant suppression of serum cytokines was observed. However, in the liver lysate of IL-37-expressing mice, IL-6 was significantly lower than that in control mice $24 \mathrm{hrs}$ after ConA challenge.

In conclusion, in vivo expression of human IL-37 in mice reduces local and systemic inflammation in ConA-induced experimental hepatitis and LPS-induced sepsis. Since transiently expressed, IL-37 is not specifically expressed in immune cells of the liver, this observation supports the in vitro generated hypothesis of IL-37 acting as an anti-inflammatory cytokine in the extracellular compartment beside its intracellular functionality.

\section{Abbreviations}

ConA: Concanavalin A

ALT: Serum alanine aminotransferase

LPS: Lipopolysaccharide.

\section{CONFLICT OF INTERESTS}

The authors declare that there is no conflict of interests with the current work. 


\section{ACKNOWLEDGMENTS}

The Authors are grateful to Andrea Sendelhofert for excellent technical assistance establishing immunohistochemical staining against IL-37. This work was supported by the Deutsche Forschungsgemeinschaft to P. Bufler (BU 1222/3-2 and BU 1222/3-3) and to R. Kappler (KA 2274/3-1).

\section{REFERENCES}

[1] C. A. Dinarello, "Why not treat human cancer with interleukin-1 blockade?" Cancer and Metastasis Reviews, vol. 29, no. 2, pp. 317-329, 2010.

[2] M. F. Nold, C. A. Nold-Petry, J. A. Zepp, B. E. Palmer, P. Bufler, and C. A. Dinarello, "IL-37 is a fundamental inhibitor of innate immunity," Nature Immunology, vol. 11, no. 11, pp. 1014-1022, 2010.

[3] S. J. Busfield, C. A. Comrack, G. Yu et al., "Identification and gene organization of three novel members of the IL-1 family on human chromosome 2," Genomics, vol. 66, no. 2, pp. 213-216, 2000.

[4] S. Kumar, P. C. McDonnell, R. Lehr et al., "Identification and initial characterization of four novel members of the interleukin-1 family," Journal of Biological Chemistry, vol. 275, no. 14, pp. 10308-10314, 2000.

[5] V. P. Smith and A. Alcami, "Expression of secreted cytokine and chemokine inhibitors by ectromelia virus," Journal of Virology, vol. 74, no. 18, pp. 8460-8471, 2000.

[6] S. L. Taylor, B. R. Renshaw, K. E. Garka, D. E. Smith, and J. E. Sims, "Genomic organization of the interleukin-1 locus," Genomics, vol. 79, no. 5, pp. 726-733, 2002.

[7] S. Kumar, C. R. Hanning, M. R. Brigham-Burke et al., "Interleukin-1F7b (IL-1H4/IL-1F7) is processed by caspase-1 and mature IL-1F7b binds to the IL-18 receptor but does not induce IFN- $\gamma$ production," Cytokine, vol. 18, no. 2, pp. 61-71, 2002.

[8] G. Pan, P. Risser, W. Mao et al., "IL-1H, an interleukin 1-related protein that binds IL-18 receptor/IL-1Rrp," Cytokine, vol. 13, no. 1, pp. 1-7, 2001.

[9] P. Bufler, T. Azam, F. Gamboni-Robertson et al., "A complex of the IL-1 homologue IL-1F7b and IL-18-binding protein reduces IL-18 activity," Proceedings of the National Academy of Sciences of the United States of America, vol. 99, no. 21, pp. 13723-13728, 2002.

[10] P. Bufler, F. Gamboni-Robertson, T. Azam, S. H. Kim, and C. A. Dinarello, "Interleukin-1 homologues IL-1F7b and IL-18 contain functional mRNA instability elements within the coding region responsive to lipopolysaccharide," Biochemical Journal, vol. 381, no. 2, pp. 503-510, 2004.

[11] G. Tiegs, J. Hentschel, and A. Wendel, "A T cell-dependent experimental liver injury in mice inducible by concanavalin A," Journal of Clinical Investigation, vol. 90, no. 1, pp. 196-203, 1992.

[12] D. S. Pratt and M. M. Kaplan, "Evaluation of abnormal liver-enzyme results in asymptomatic patients," New England Journal of Medicine, vol. 342, no. 17, pp. 1266-1271, 2000.

[13] F. Gantner, M. Leist, A. W. Lohse, P. G. Germann, and G. Tiegs, "Concanavalin A-induced T-cell-mediated hepatic injury in mice: the role of tumor necrosis factor," Hepatology, vol. 21, no. 1, pp. 190-198, 1995.

[14] K. Takeda, Y. Hayakawa, L. Van Kaer, H. Matsuda, H. Yagita, and K. Okumura, "Critical contribution of liver natural killer T cells to a murine model of hepatitis," Proceedings of the National Academy of Sciences of the United States of America, vol. 97, no. 10, pp. 5498-5503, 2000.

[15] B. Jaruga, F. Hong, R. Sun, S. Radaeva, and B. Gao, "Crucial role of IL-4/STAT6 in T cell-mediated hepatitis: upregulating eotaxins and IL-5 and recruiting leukocytes," Journal of Immunology, vol. 171, no. 6, pp. 3233-3244, 2003.

[16] S. Kusters, F. Gantner, G. Kunstle, and G. Tiegs, "Interferon gamma plays a critical role in T cell-dependent liver injury in mice initiated by concanavalin A," Gastroenterology, vol. 111, no. 2, pp. 462-471, 1996.

[17] H. Tsutsui, N. Kayagaki, K. Kuida et al., "Caspase-1-independent, Fas/Fas ligand-mediated IL-18 secretion from macrophages causes acute liver injury in mice," Immunity, vol. 11, no. 3, pp. 359-367, 1999.

[18] G. Sass, K. Koerber, and G. Tiegs, "TNF tolerance and cytotoxicity in the liver: the role of interleukin$1 \beta$, inducible nitric oxide-synthase and heme oxygenase-1 in D-galactosamine-sensitized mice," Inflammation Research, vol. 51, no. 5, pp. 229-235, 2002. 
[19] H. Mizuhara, M. Uno, N. Seki et al., "Critical involvement of interferon gamma in the pathogenesis of T-cell activation-associated hepatitis and regulatory mechanisms of interleukin-6 for the manifestations of hepatitis," Hepatology, vol. 23, no. 6, pp. 1608-1615, 1996.

[20] H. Louis, O. Le Moine, M. Goldman, and J. Devière, "Modulation of liver injury by interleukin-10," Acta GastroEnterologica Belgica, vol. 66, no. 1, pp. 7-14, 2003.

[21] K. L. Streetz, T. Wüstefeld, C. Klein, M. P. Manns, and C. Trautwein, "Mediators of inflammation and acute phase response in the liver," Cellular and Molecular Biology, vol. 47, no. 4, pp. 661-673, 2001.

[22] H. Mizuhara, E. O'Neill, N. Seki et al., "T cell activation-associated hepatic injury: mediation by tumor necrosis factors and protection by interleukin 6," Journal of Experimental Medicine, vol. 179, no. 5, pp. 1529-1537, 1994.

[23] S. Radaeva, R. Sun, H. N. Pan, F. Hong, and B. Gao, "Interleukin 22 (IL-22) plays a protective role in T cellmediated murine hepatitis: IL-22 Is a survival factor for hepatocytes via STAT3 activation," Hepatology, vol. 39, no. 5, pp. 1332-1342, 2004.

[24] F. Liu, Y. K. Song, and D. Liu, "Hydrodynamics-based transfection in animals by systemic administration of plasmid DNA," Gene Therapy, vol. 6, no. 7, pp. 1258-1266, 1999.

[25] C. H. Contag, P. R. Contag, J. I. Mullins, S. D. Spilman, and D. A. Benaron, "Photonic detection of bacterial pathogens in living hosts," Molecular Microbiology, vol. 18, no. 4, pp. 593-603, 1995.

[26] B. Siegmund, K. C. Lear-Kaul, R. Faggioni, and G. Fantuzzi, "Leptin deficiency, not obesity, protects mice from Con-A-induced hepatitis," European Journal of Immunology, vol. 32, no. 2, pp. 552-560, 2002.

[27] I. Bohlinger, M. Leist, F. Gantner, S. Angermüller, G. Tiegs, and A. Wendel, "DNA fragmentation in mouse organs during endotoxic shock," American Journal of Pathology, vol. 149, no. 4, pp. 1381-1393, 1996.

[28] S. Sharma, N. Kulk, M. F. Nold et al., "The IL-1 family member 7b translocates to the nucleus and down-regulates proinflammatory cytokines," Journal of Immunology, vol. 180, no. 8, pp. 5477-5482, 2008.

[29] F. Andrianaivo, M. Lecocq, S. Wattiaux-De Coninck, R. Wattiaux, and M. Jadot, "Hydrodynamics-based transfection of the liver: entrance into hepatocytes of DNA that causes expression takes place very early after injection," Journal of Gene Medicine, vol. 6, no. 8, pp. 877-883, 2004.

[30] D. M. Feng, C. X. He, C. Y. Miao et al., "Conditions affecting hydrodynamics-based gene delivery into mouse liver in vivo," Clinical and Experimental Pharmacology and Physiology, vol. 31, no. 12, pp. 850-855, 2004.

[31] J. Schümann, D. Wolf, A. Pahl et al., "Importance of Kupffer cells for T-cell-dependent liver injury in mice," American Journal of Pathology, vol. 157, no. 5, pp. 1671-1683, 2000.

\section{This article should be cited as follows:}

Ana-Maria Bulau, Michaela Fink, Christof Maucksch, Roland Kappler, Doris Mayr, Kai Wagner, and Philip Bufler, "In Vivo Expression of Interleukin-37 Reduces Local and Systemic Inflammation in Concanavalin A-Induced Hepatitis," TheScientificWorldJOURNAL, vol. 11, pp. 2480-2490, 2011. 


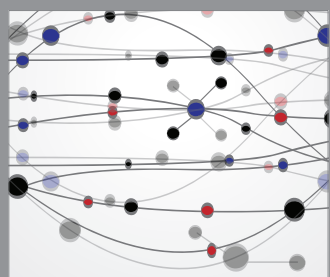

The Scientific World Journal
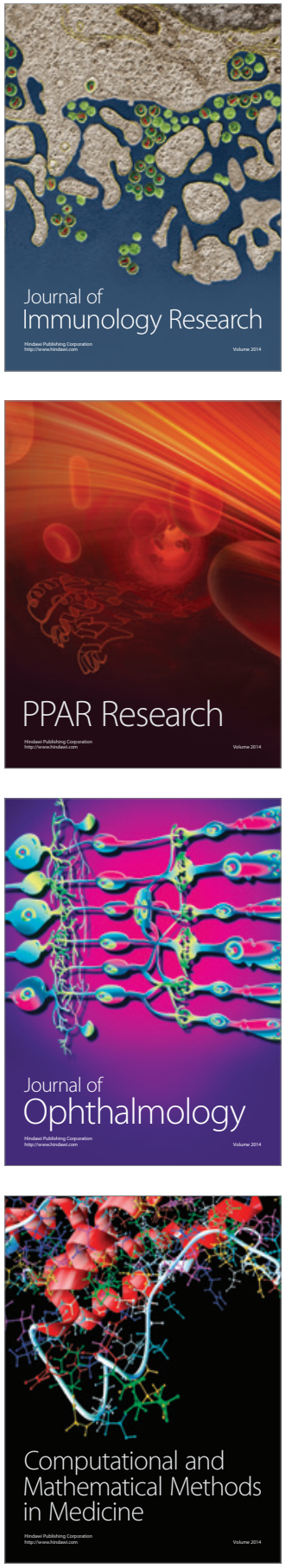

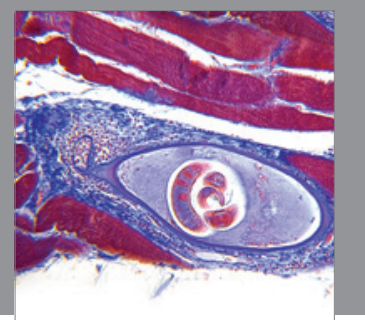

Gastroenterology

Research and Practice
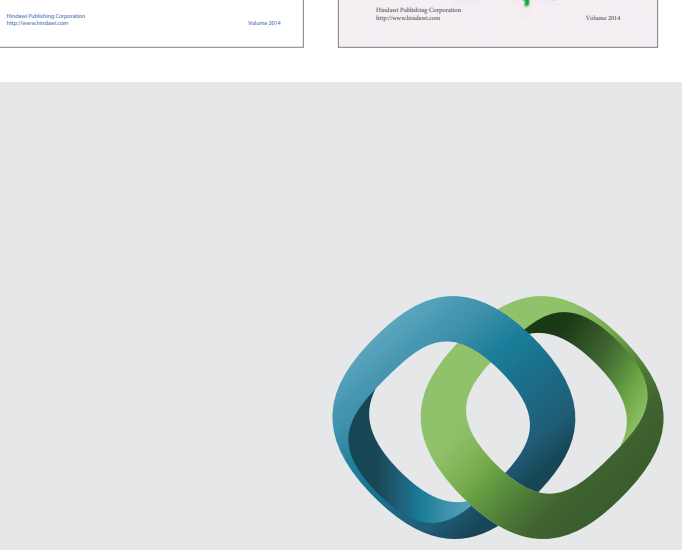

\section{Hindawi}

Submit your manuscripts at

http://www.hindawi.com
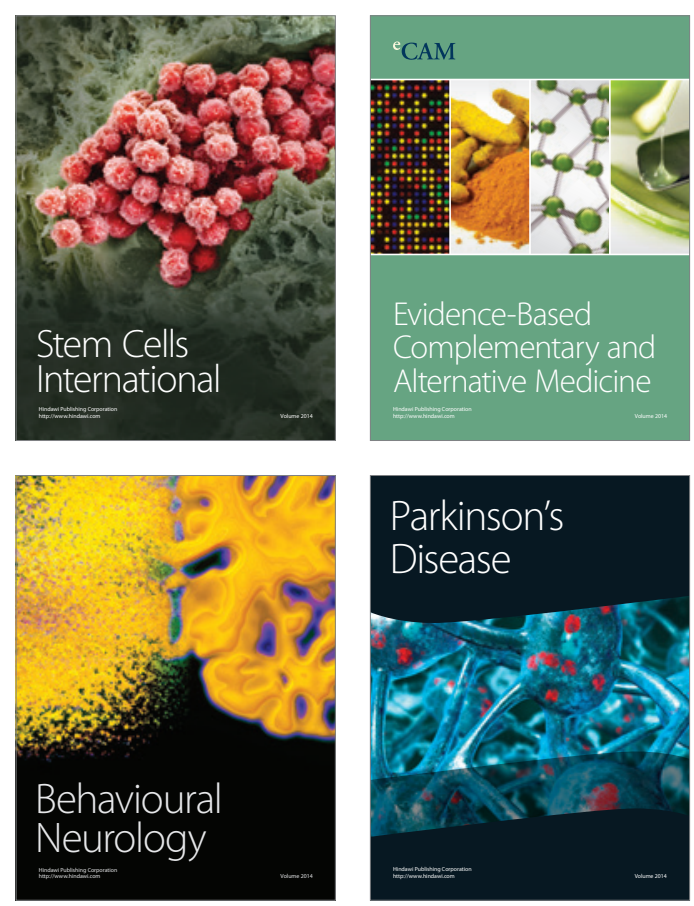

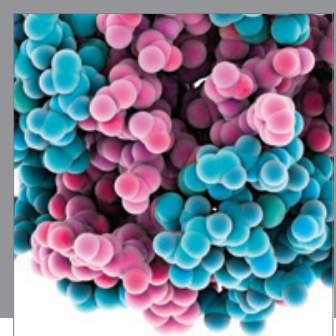

Journal of
Diabetes Research

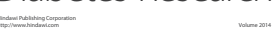

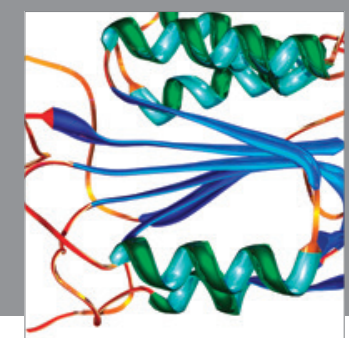

Disease Markers
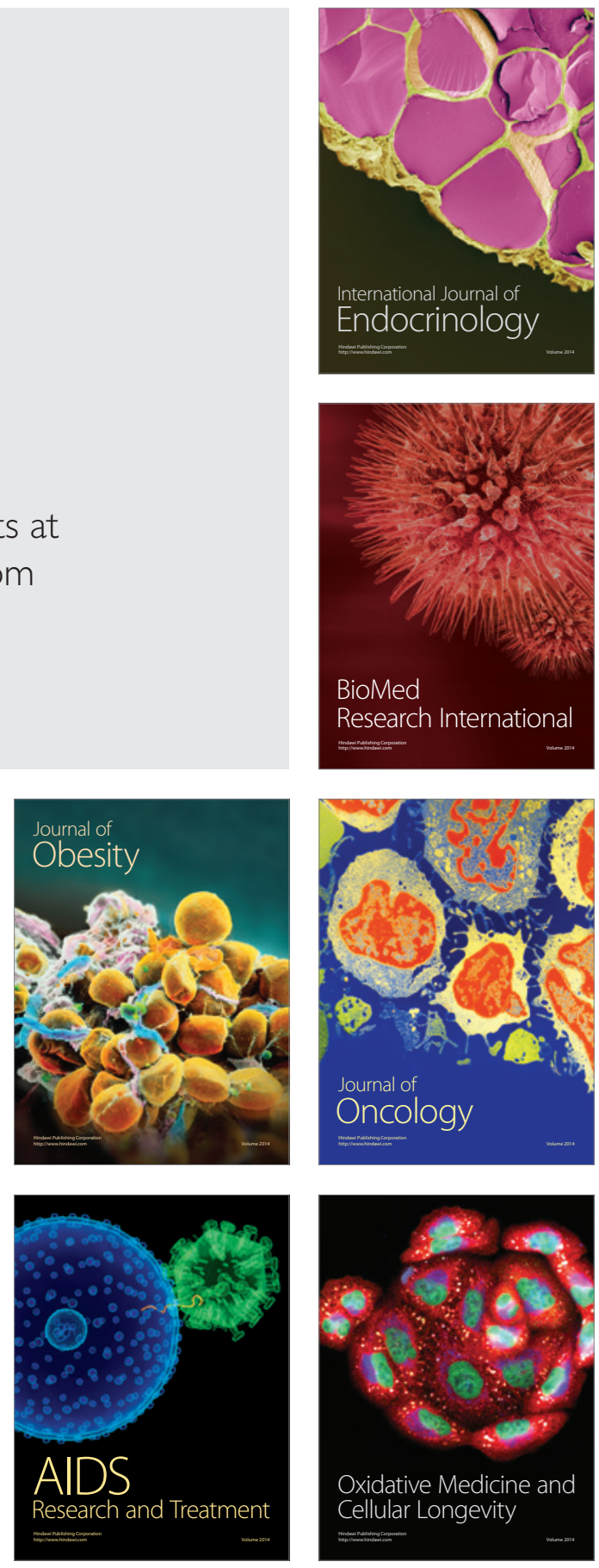